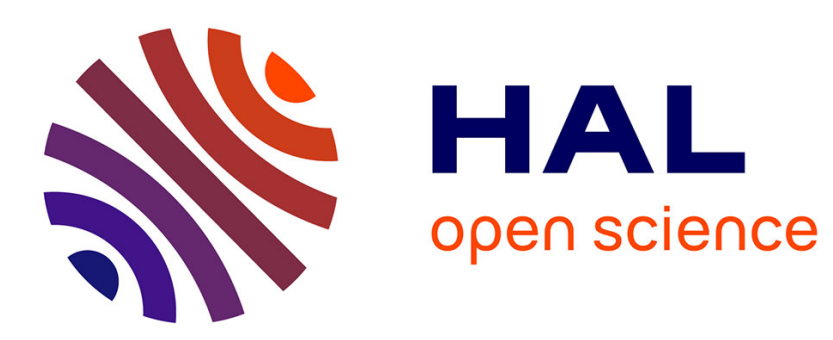

\title{
STRUCTURE DES JOINTS EN MICROSCOPIE ELECTRONIQUE HAUTE RESOLUTION (MEHR)
}

\author{
A. Bourret, C. d'Anterroches, J. Penisson
}

\section{To cite this version:}

A. Bourret, C. d'Anterroches, J. Penisson. STRUCTURE DES JOINTS EN MICROSCOPIE ELECTRONIQUE HAUTE RESOLUTION (MEHR). Journal de Physique Colloques, 1982, 43 (C6), pp.C683-C6-92. 10.1051/jphyscol:1982609 . jpa-00222289

\section{HAL Id: jpa-00222289 https://hal.science/jpa-00222289}

Submitted on 1 Jan 1982

HAL is a multi-disciplinary open access archive for the deposit and dissemination of scientific research documents, whether they are published or not. The documents may come from teaching and research institutions in France or abroad, or from public or private research centers.
L'archive ouverte pluridisciplinaire HAL, est destinée au dépôt et à la diffusion de documents scientifiques de niveau recherche, publiés ou non, émanant des établissements d'enseignement et de recherche français ou étrangers, des laboratoires publics ou privés. 
JOURNAL DE PHYSIQUE

Cozzoque C6, supplément au $n^{\circ} 12$, Tome 43, décembre 1982

page $66-83$

\title{
STRUCTURE DES JOINTS EN MICROSCOPIE ELECTRONIQUE HAUTE RESOLUTION (MEHR)
}

\author{
A. Bourret, C. d'Anterroches* et J.M. Penisson \\ Centre d'Etudes Nucléaires de Grenoble, Département de Recherche Fondamentale, \\ Section de Physique du Solide, 85X,38041 Grenobie Cedex, France
}

\begin{abstract}
Résumé - La microscopie électronique haute rẻsolution (MEHR) permet dans des conditions gẻométriques particulières l'observation directe des structures au voisinage des joints de grains. Ces conditions sont limitées aux joints de flexion d'axe commun parallèle à une direction dense du cristal. Cette technique a été empjoyée sur plusieurs macies dans le germanium et le molybdène $(\Sigma 41, \Sigma 25, \Sigma 9$ et $\Sigma 3)$. Les relaxations primaires et secondaires. ont été mises en êvidence et les vecteurs de Burgers des primaires mesurés. Une discussion des possibilités et des limites de la méthode est prësentēe.
\end{abstract}

\begin{abstract}
The high resolution electron microscopy (HREM) allows the direct observation of the grain boundary (GB) microstructure in favorable cases. The G.B. must be pure tilt with a dense axis as a common direction. This technique has been employed on several twins in germanium and molybdenum ( $\Sigma 41, \Sigma 25, \Sigma 9$ and $\Sigma 3$ ). Primary and secondary relaxations have been imaged and Burgers vector of primary dislocations measured. Possibilities and limits of the HREM are discussed.
\end{abstract}

1. Introduction - Jusqu'à récemment l'étude des joints de grains par microscopie èlectronique haute résolution (MEHR) était limitée aux semiconducteurs dans la seule direction cristallographique dense [011] (1) (9). Dans les métaux ou pour des directions cristallographiques différentes les études se faisaient soit par T'utilisation du faisceau incliné (2) soit par l'utilisation de tensions très êlevēes (3). Le dêveloppement de microscopes à $200 \mathrm{keV}$ devait permettre, avec une résolution proche de $0.2 \mathrm{~nm}$, d'accéder aux structures atomiques des interfaces. De plus la possibilité d'obtention de bicristaux de caractéristiques prédéterminées autorisait la comparaison des structures observées aux modèles existants. Les matêriaux étudiés dans le présent travail sont respectivement le germanium et le silicium cubique diamant et le molybdène (cubique centré). Les axes d'observation sont [011] et [001] pour Ge et $\mathrm{Si}$ et [001] pour Mo. Pour ces joints nous avons essayé de préciser la nature périodique ou non des interfaces, la valeur des relaxations rigides ou locales et la nature physique des relaxations secondaires au voisinage des position de macle.

2. La méthode de M.E.H.R. - La MEHR effectuée à 100 ou à $200 \mathrm{keV}$ permet avec les meilleures piēces potaires objectif actuelles d'accéder respectivement à des rêsolutions structurellement interprétables de $0.3 \mathrm{~nm}$ et $0.2 \mathrm{~nm}$. De plus une image de microscopie étant limitée à une projection selon l'axe d.'observation de la structure il est absolument nécessaire pour obtenir des informations utiles d'utiliser des directions denses du cristal. Selon ces directions tous les atomes ou groupes d'atomes s'alignent pour donner un seul point en projection et ces points seront ainsi les plus distants possible les uns des autres. Dans le cristal parfait les projections sont donc extrêmement limitêes. Dans le cas du cubique diamant la structure se projette facilement selon [011] : cependant chaque tache dans 1 'image représente un doublet d'atomes indiscernables (8). Selon [007], et seulement à $200 \mathrm{keV}$, il est possible de distinguer la projection de chaque colonne

* Nouvelle adresse : CNET-CNS - BP 42 - 38240 MEYLAN (France) 
atomique, aussi bien pour Ge que pour Mo. Dans le cas d'un joint de grain la structure pour être lisible doit être invariante le long de l'axe d'observation. Ceci limite ainsi l'application de la MEHR aux joints de flexion pure autour d'axes communs simples et selon lesquels la structure sera observée.

$L$ 'interprétation complète du contraste des images dans les zones non périodiques ( 1 'interface et ses relaxations locales) est assez complexe et dépend beaucoup des distances dans l'objet comparées à la résolution. Le contraste d'un objet donné dépend essentiellement de la défocalisation et de l'épaisseur d'échantillons traversêe. Aussi pour relier correctement image et objet la connaissance expérimentale de ces deux paramètres est-elle nécessaire. De plus le lien entre le potentiel projeté ét l'intensitê dans l'image n'est pas linēaire,bien que connu, mais surtout $n$ 'est pas inversible (on ne peut remonter directement de $l^{\prime}$ image à l'objet). Cependant c'est précisément la structure cristalline donc la position des atomes projetés que 1 'on veut déduire au voisinage d'un joint à partir de l'image. Pour cela il est donc nécessaire de simuler complètement (ceci se fait sur ordinateur) l'image à partir d'une structure hypothétique puis de corriger cette dernière jusqu'à ce que les images simulées et expérimentales concordent (4).

Les précision obtenues par cette méthode pour une rêsolution de $0.2 \mathrm{~nm}$ ou voisine sont les suivantes (5) : la précision sur le positionnement d'une colonne atomique (ou d'une paire dans le cas du Ge) est de 1'ordre de $14 \%$ de la résolution soit $0.02 \mathrm{~nm}$. De plus la détection de la présence (ou de l'absence) d'une colonne atomique dans les structures de coeur dépend beaucoup de la distance (projetée) aux colonnes les plus proches : pour toute distance supérieure à la résolution la colonne est détectable, dans le cas inverse ce n'est généralement pas possible. Enfin il est difficile de distinguer si une colonne atomique est partiellement occupée ou non la limite de détection étant de l'ordre de $50 \%$ d'une colonne. Enfin $i l$ faut encore souligner que toute information le long de l'axe de projection est perdue dans la plupart des cas. Malgré ces différentes limitations la méthode MEHR permet d'extraire des informations d'ordre très localccontrairement aux méthodes de diffraction qui intègrentsur une grande distance. Cette mêthode sera donc particuliêrement adaptée à l'étude des interfaces complexes, non périodiques présentant des dislocations ou discontinuités nombreuses. Nous verrons cependant que les positions de macle à haute coincidence et symétriques nous ont fourni un certain nombre d'objets tests pour la méthode et nous ont permis d'aborder l'étude de positions de macles d'indices plus élevés.

3. Conditions expérimentales - La MEHR est effectuée sur un microscope JEOL $100 \mathrm{C}$, et un $200 \mathrm{CX}$ en utilisant une pièce polaire objectif de $C_{S}=0.7 \mathrm{~mm}$ à $100 \mathrm{keV}$ et $1.1 \mathrm{~mm} a ̀ ~ 200 \mathrm{keV}$. L'image est formée en faisceau non inclinê et en conditions de diffraction de Laủe symétriques : 9 faisceaux sont en gênéral inclus dans le diaphragme de 1 'objectif pour former 7 'image. Ces faisceaux sont du type $<111>$ pour chaque cristal dans le cas d'un axe [011] et du type $\langle 200\rangle$ ou $\langle 110\rangle$ dans le cas d'un axe [001]. Les clichés sont ensuite exp̄loitēs quantitativement sur banc optique pour dēterminer la distance de défocalisation.; deux dēfocalisations principales sont utilisées:la défocalisation de Scherzer $(-50 \mathrm{~nm})$ et la défocalisation donnant le contrast inverse $\sim(-90 \mathrm{~nm})$. Pour la défocalisation de Scherzer les atomes sont nöirs et donc blancs pour l'autre défocalisation. Pour imager les périodicitês $0.2 \mathrm{~nm}$ une défocalisation encore plus grande est nécessaire ( $120 \mathrm{~nm}$ ) qui rend les atomes noirs. L'épaisseur de l'échantilion est évaluêe à partir de la localisation des franges de Pendellösung.

La périodicité de l'interface est observée soit directement sur 1 'image soit sur un diffractogramme optique. La projection du vecteur de Burgers des dislocations primaires est obtenue directement en traçant un circuit de Burgers : ceci suppose qu'il soit possible de trouver une zone continue permettant de passer d'un cristal à l'autre à la traversée de l'interface. Les relaxations secondaires dans le cas où elles existent, sont détectées par la présence le long de l'interface de modifications du motif de base périodique de la macle. Les translations rigides sont mesurées au moyen de grilles transparentes mises en coincidence sur les deux parties cristallines de part et d'autre du joint : dans la région de recouvrement de ces grilles les coincidences et les translations éventuelles apparaissent. Cependant cette méthode est peu précise (0.02 nm environ) et si elle est utile pour 
trouver le type de coincidence dans le cas des semiconducteurs, elle n'a pu rêvéler les faibles translations détectées par la méthode des franges $\alpha$. Les relaxations atomiques enfin sont mesurées par comparaison avec des images simulées. Ces simulations sont effectuées sur un calculateur Plurimat $S$ spécialisé : les images calculées comportent $128 \times 128$ points avec une résolution de l'ordre de $0.02 \mathrm{~nm}$. Les joints de grains dans le Ge sont produits par la méthode de Czochralski (6) en utilisant les germes orientés. Les principales impuretés sont l'oxygène et le carbone ( $10^{17}$ at $\mathrm{cm}^{3}$ ). Par contre les bicristaux de molybdène sont préparés par collage sous faisceau d'électrons (7) : pour éviter toute ségrégation fragilisante de l'oxygène ceux-ci sont recuits $8 \mathrm{~h}$ à $2000^{\circ} \mathrm{C}$ sous ultra vide : malgré ce traitement les joints restent fragiles et 1 'oxygène $n$ 'est certainement pas complétement êliminé.

4. Les joints d'axe commun [011] (germanium et silicium) - La macle miroir $\sum 3$ (ī1ī) est un excellent objet test que nous avons utilisê pour Ge et Si. En effet $1 \mathrm{a}$ position de coincidence est telle que le plan de symétrie passe entre les atomes $c^{\prime}$ est-à-dire entre deux plans atomiques $\{111\}$. Pour la défocalisation qui rend les atomes blancs : le plan miroir est alors entre deux rangées de points blancs. Réciproquement pour la défocalisation qui rend les atomes noirs ce plan miroir passe sur une rangẻe de points blancs : dans ces conditions se sont les tunnels de la structure qui sont images comme des points blancs (fig. 1). L'addition d'une dẻsorientation supplêmentaire à la macle $\Sigma 3$ (IIII) de $1^{\circ}$ produit une sêrie de dislocations d'interface de vecteur de Burgers $1 / 3$ [ $\overline{1} \overline{1} \overline{1}]$ analogue à celle produite dans un sous joint. Ces dislocations dont le vecteur de Burgers est un vecteur du réseau dsc de la macle correspondante se prêsentent sous deux formes (fig. 1). Une forme non dissociée (fig. la, b) qui est la forme classique de la dislocation d'interface : un modèle directement issu des images peut être construit. Le coeur comporte un cycle à 5 atomes et un cycle à 8 branches avec une reconstruction probable en $r$ similaire à celle imaginêe pour la partielle $90^{\circ}$ (14). La forme
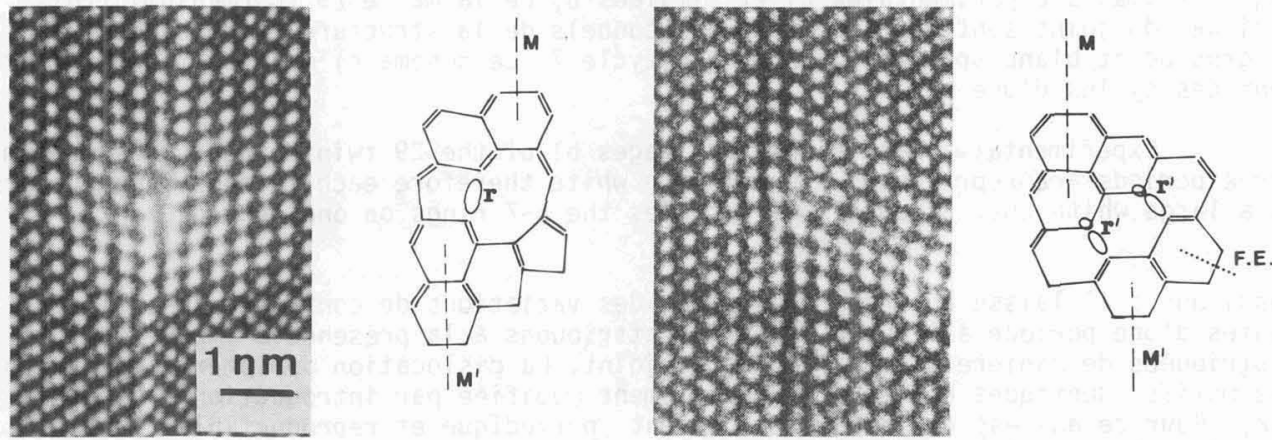

Fig. 1 - Les dislocations "secondaires" associēes à un joint $\Sigma 3+1^{\circ}$. a) dislocation de Frank b) le schêma atomique correspondant c) dislecation dissociée d) le schéma atomique correspondant à la stair-rod restant dans le joint. Silicium - les atomes sont noirs en a et blancs en $c$.

Secondary dislocations in a $\Sigma 3+1^{\circ} \mathrm{GB}$ a) Frank dislocation b) corresponding atomic pattern c) dissociated dislocation d) atomic pattern of the stair-rod remaining in the GB. Silicon atoms are dark in a) and white in c).

dissociêe (fig. 1c-d) émet à partir du joint une dislocation de Schokley glissile $1 / 6$ [21 1$]$ laissant dans le joint une dislocation "stair-rod" $1 / 6$ [017]. Le coeur de cette dislocation est complexe et contient des liaisons dont la reconstruction est facile à imaginer. Un arrangement presque similaire se retrouve dans les intersections de fautes d'empilement classiques : dans ce cas les reconstructions se font par doublement de la périodicité le long de l'axe [011].

La macle $\Sigma 9$ (122) présente également une périodicité et des symétries remarquables qui ont étê retrouvées par microscopie électronique. Cette macle déjà observêe par Krivanek et al (1) a une période de $1,2 \mathrm{~nm}$ et un vecteur de Burgers global de 
[01ī] sur une période. En fait l'arrangement des atomes constitue une sêrie de 2 cycles à 5 et 7 atomes imbriqués. Chaque groupement 5-7 est identique à celui présent sur une dislocation coin de Lomer : ceci correspond au vieux modèle de Hornstra (10). Ces images expérimentales ont èté compárées en détail (11) avec les images simulées à partir d'un modèle similaire à celui de Hornstra. Le modēle est défini par une coincidence exacte de type $C_{2}$ (atome 000 du cristal I coincidant avec atome 1/4 1/4 1/4 du cristal II) sans aucune relaxation locale : images simulées et expérimentales coincident très bien lorsque les atomes sont noirs (fig. 2). Pour une défocalisation donnant le contraste inverse l'accord n'est pas
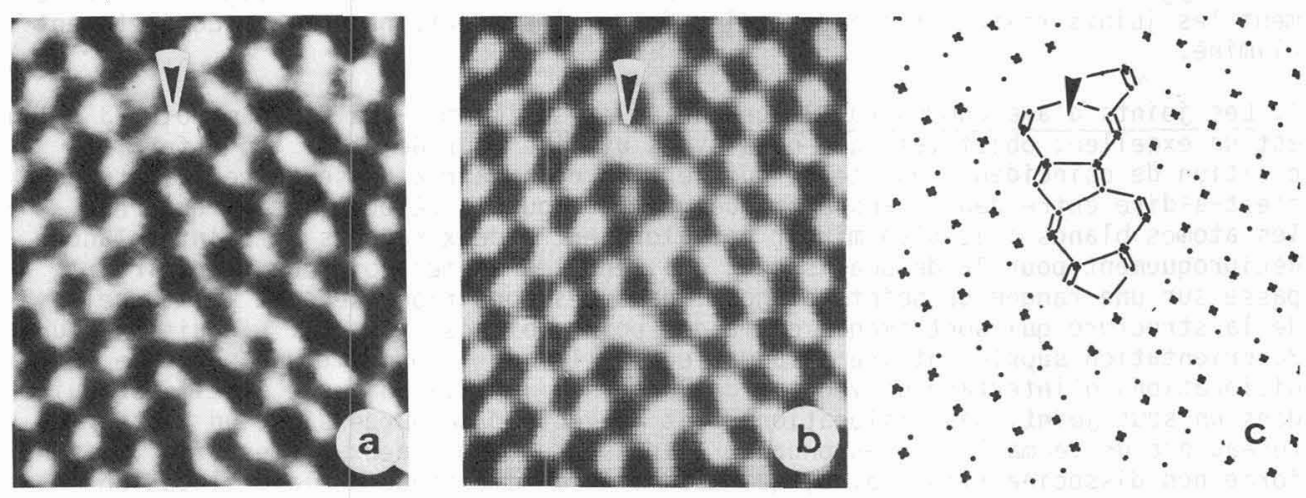

Fig. 2 - Images expérimentales a) et simulées b) de la macle $\Sigma 9$ (germanium) trois périodes du joint sont représentées. Les tunnels de la structure étant blancs, un gros point blanc apparaît dans chaque cycle 7 . Le schëma c) montre 1 'enchainement des cycles d'une période.

Experimental a) and simulated images b) of the $\Sigma 9$ twin boundary (germanium). three periods are represented. Tunnels are white therefore each 7 atoms ring appears as a large white dot. Scheme c) illustrates the 5-7 rings on one period.

aussi bon : $i 1$ Taisse apparaître en effet des variations de contraste assez importantes d'une période à l'autre. Nous les attribuons à la présence d'impuretēs distribuées de manière alēatoire dans le joint. La dislocation de Lomer comportant des motifs identiques est en effet facilement modifiêe par introduction d'oxygène (12). Pour ce qui est de la partie vraiment périodique et reproductible d'une période à l'autre les relaxations locales pour ce joint sont inférieures à $0.02 \mathrm{~nm}$. On remarquera que dans ce modèle les angles des liaisons covalentes sont fortement perturbēs sur certains cycles pouvant atteindre $140^{\circ}$ au lieu de $114^{\circ}$; il en est de même pour les longueurs. Dans le cas d'une dislocation de Lomer avec cyctes 5-7 les longueurs et les angles sont moins affectēs.

A titre d'exemple nous présentons maintenant un joint d'angle plus faible et présentant asymétrie et facettage. Ces configurations sont très fréquentes dans les polycristaux et illustrent bien l'intérêt de la MEHR pour ces joints non périodiques. L'image d'un tel joint (fig. 3 ) d'angle $10^{\circ}$ est d'une grande complexité. Le joint contient en effet une sêrie de portions de structure différente. Les dislocations primaires ont des vecteurs de Burgers égaux à $\left.\left.b_{A}=1 / 2<110\right\rangle b_{B}=1 / 2<112\right\rangle b_{C}=\langle 111\rangle$. Ces différentes portions peuvent donc $s^{\prime}$ analyser comme des joints de plans diffêrents produisant un facettage complexe. Ces dislocations qui apparajssent également dans les sous joints $\left(\lesssim 1^{\circ}\right)$ en se mélangeant permettent d'introduire une grande variété de joints non périodiques et asymétriques. On voit également que l'introduction de motifs de base rencontrés dans les dislocations des sous joints peut servir à construire les joints d'angle 


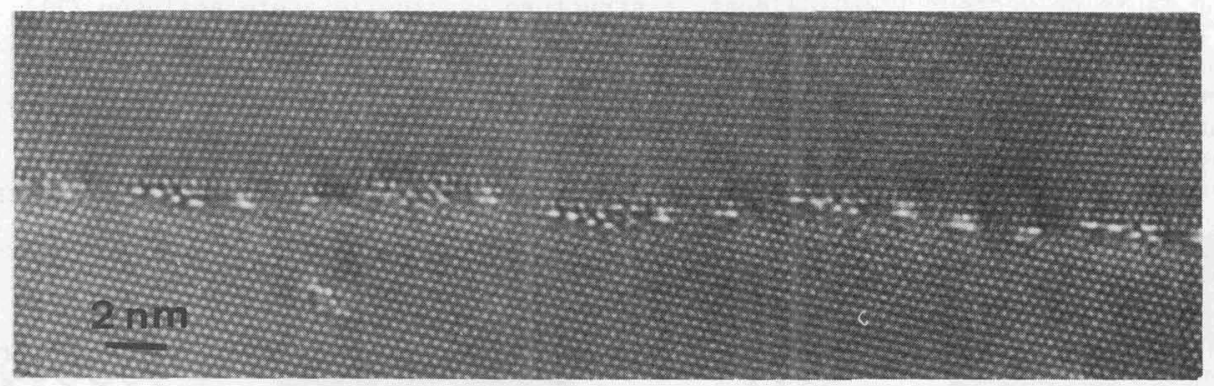

Fig. 3 - Joint de flexion de $10^{\circ}$ axe commun [011] dans le germanium. Ce joint contient 4 types de dislocations dont les vecteurs de Burgers mesurés directement sur le clichể sont diffêrents.

Pure tilt $G B\left(\theta=10^{\circ}\right)$ [011] common axis in germanium. This boundary is formed by a complex combination of four dislocations. The directly measured vector are : $A=b_{A}=1 / 2\langle 110\rangle ; B=b_{B}=1 / 2\langle 112\rangle ; C=b_{C}=\langle 111\rangle$

plus élevé et de plans de joint très divers. Comme nous avons pu le voir pour le motif d'une dislocation de Frank dans $\Sigma 3$ ces motifs de base sont souvent. complexes et comprennent des réarrangements de liaisons très différents de l'arrangement têtraédrique simple.

De même les observations effectuées sur $\Sigma 27$ asymétrique par Cunningham et Ast montrent que les angles des facettes font apparaitre des structures dont les liaisons sont compliquées.

I1 faut enfin remarquer qu'en général le long de [011] les reconstructions sont de deux types (14) : elles peuvent se faire sans destruction de la périodicitê selon [011] ou en faisant apparaître une périodicité double. I] serait intēressant de mettre en évidence ces périodicités doubles dans les joints d'axe [011] ma is pour l'instant ceci n'a pu être fait.

5. Les joints d'axe commun [001] germanium et molybdène - Notre étude a porté sur des joints de macle d'angle assez faible et deux matériaux de structure différente. Les résultats obtenus sur ces deux corps pour la même macle $\Sigma 41$ sont intêressants à comparer.

Dans le cas du germanium les macles $\Sigma 25$ et $\Sigma 41$ de plans respectifs (1770) et (i90) ont été observées : elles présentent des caractēristiques tout à fait similaires. Il est aisé en particulier de définir par un circuit de Burgers approprié les vecteurs de Burgers des dislocations. Celles ci sont suffisamment éloignées pour que les zones de continuité à la traversée du cristal soient faciles à trouver. La périodicité observêe est de $2.0 \mathrm{~nm}$ et $2.55 \mathrm{~nm}$ ce qui correspond à la périodicité du réseau de coincidence (cs1) dans ce plan, et les dislocations sont de vecteur globaux $\vec{b}_{\text {projeté }}=[010]$. Cependant (fig. 4a) te coeur de ces dislocations s'étend sur plusieurs distances atomiques et nous avons une dissociation nette en deux parties. La distance exacte entre ces deux parties est difficile à déterminer avec la résolution actuelle. Elle paraît se situer entre 0.5 et $0.8 \mathrm{~nm}$ et demeure toujours plus faible que la moitié de la période. De ce fait la périodicité du csl est nettement observée : elle est d'ailleurs soulignée par la légère attaque chimique qui a lieu au voisinage du groupement des deux dislocations associées.

La composante des vecteurs de Burgers parallè]e à l'axe d'observation [001] n'étant pas accessible à l'expérience il y a a priori deux modèles possibles :

(1) $b_{p}=[010] \rightarrow 1 / 2[010]+1 / 2[010]$ sêparés par une faute d'empilement

(2) ou $[010] \rightarrow 1 / 2[011]+1 / 2[011]$ 
Dans la lère hypothèse les dislocations sont imparfaites et sont séparées par une faute de vecteur 1/2[010] dont la structure éventuelle a été analysêe (11). L'arrangement proposé ne maintient pas la structure trétracoordonnée et devrait donc être très instable; de plus la continuité des plans atomiques entre les deux dislocations est très nette expérimentalement ce qui indique l'absence de faute. Nous retiendrons donc la dissociation (2) car il y a une translation rigide le Tong de 7 'axe [001] (Bacmann communication privée). Dans le molybdène (fig. 4b) le joint comporte par rapport à la macle $\Sigma 41$ un écart
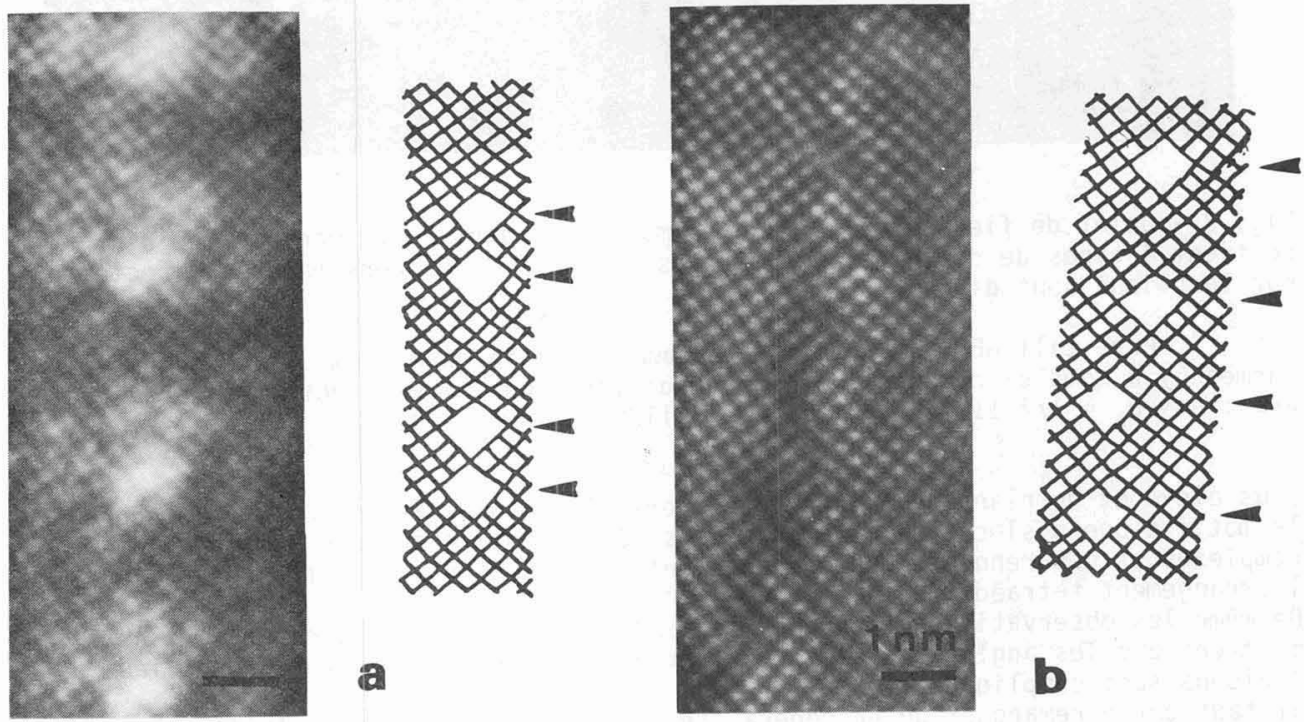

Fig. 4 - Joints de grains d'orientation voisine de $\Sigma 41$ a) germanium b) molybdène. Les plans supplémentaires marquant l'emplacement des dislocations sont indiquées.

$\Sigma 41$ grain boundary in a) germanium and b) molybdène. Extra half plane indicating dislocation location are outlined.

assez important (16). Si nous considérons pour l'instant dans la structure facettēe qui en rësulte, uniquement les parties correspondant à la coincidence exacte $\Sigma 41$ nous avons une sërie de dislocations de périodicité $1.4 \mathrm{~nm}$ de vecteur de Burgers [010] c'est-à-dire purement coịn. La périodicité apparente est 1a moitié de celle du csl puisque les coincidences en 000 et 111 qui apparaissent successivement ne sont pas distinguables. La dislocation [010] n'est pas dissociée. Si nous comparons maintenant germanium et molybdène nous voyons deux diffërences importantes : la périodicité du csl est révêlée dans le cas du cubique diamant alors qu'on a une périodicitē moitié dans le cas du cubique centrê. De plus malgré une dissociation dans le cas du cubique diamant les images sont dissemblables en projection selon [001], car les deux dislocations provenant de la dissociation restent groupées. Ce groupement doit provenir d'un effet de coeur car élastiquement l'énergie dépend peu de la distance entre ces dislocations. La structure atomique de $\Sigma 41$ symétrique dans un cubique centrê a été calculèe par Vitek et al (17). Ces auteurs trouvent une organisation régulière de dislocations coins [010] tout à fait similaire à celle que nous avons observée.

Dans les parties asymétriques du joint (fig. 5) des dislocations additionnelles 1/2[111] s'intercalent : ces dislocations sont dues à la déviation par rapport à la position de macle. Celle-ci est une flexion pure autour de [100] d'angle $1^{\circ}$ Comme Penisson et al 7 'ont expliqué (16) cette déviation explique à la fois le facettage du joint et les types de vecteur de Burgers employēs. La troisiēme série de dislocations mixtes qui devrait accomoder la rotation mesurée (inclinée 
par rapport au deux autres) n'a pas encore pu être mise en évidence : la MEHR est impuissante à le faire et les autres méthodes sur des joints inclinês n'ont pas une assez bonne résolution.

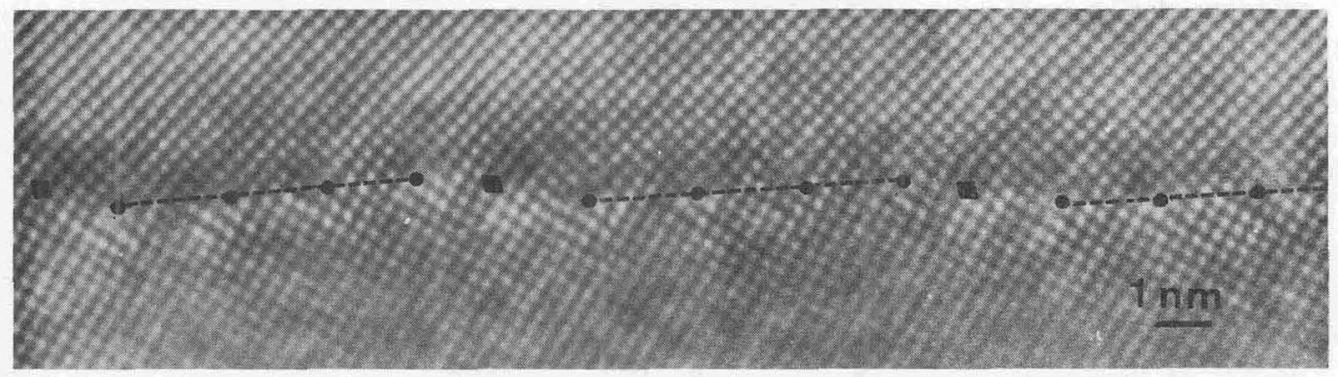

Fig. 5 - Les parties asymétriques du joint proche de $\Sigma 41$ dans le molybdène. Les dislocations additionnelles $1 / 2[111]$ sont soulignées. Remarquer le facettage régulier qui apparaît; jes facettes sont en orientation de macle $\Sigma 41$.

Asymmetrical part of a $\Sigma 41$ grain boundary in molybdenum. Additional dislocations $b=1 / 2<111>$ are outlined. Note the regular facetting with the shift of the $\Sigma 41$ twin produced by each additional dislocations.

L'exploitation quantitative de nos résultats (positionnement exact des colonnes atomiques ; détermination non ambigüe du nombre d'atomes dans les coeurs, détermination de la distance d'élargissement du coeur des dislocations) se heurte cependant à de sêrieuses difficultés dans le cas des orientations [001]. En effet les distances caractéristiques de l'objet qui doivent être bien transmises sont proches de $2 \AA$ : or ces distances sont très voisines de la résolution limite du microscope $200 \mathrm{keV}$. Plus précisément la fonction de transfert utilisēe pour imager ces distances comportent deux, ou plus fréquemment trois, oscillations. Ceci a pour effet de rendre l'image difficilement interprétable avec certitude De plus un problème expérimental non négligeable surgit pour des distances proches de la résolution limite : l'alignement optique du microscope devient primordial. or cet alignement n'est en général pas facile à rēaliser ã $200 \mathrm{keV}$ et nécessite la mise en oeuvre de techniques spéciales qui viennent seulement apparaître (18).

6. Conclusion : les possibilitês et limites de la MEHR - Les quelques exemples qui viennent d'être donnês illustrent bien les possibilités et les limites de la MEHR. Les possibilités d'études des joints de flexion autour d'axes simples sont très étendues : la méthode permet d'accéder à une information directe de caractère local. Ainsi pour toutes les structures un peu complexes comportant des microfacettages, des irrégularités, des rotations de plans de joint ou tout autre défaut perturbant la simple périodicité des joints de macle parfait cette méthode restera unique. De plus la détection des relaxations locales et la preuve directe de 1 'existence de certains motifs spécifiques des coeurs reposeront également principalement sur cette méthode. La comparaison entre les modēles de relaxation calculés et les positions déterminées expérimentalement est possible : elle demande un travail très prēcis d'exploitation des clichés. Il faut cependant rappeler que la précision actuelle de positionnement est de l'ordre de $10 \%$ de la résolution. Ainsi en utilisant les meilleurs microscopes actuellement prévus pour les années à venir la précision sera au mieux de l'ordre de $0.01 \mathrm{~nm}$. Ceci $n$ 'est pas toujours suffisant pour vérifier la validité d'un potentiel atomique ou d'un autre. La méthode devrait cependant être intéressante pour tous les cas où la densité locale d'atomes dans le joint est mal connue (coeur vide ou plein en certaines positions).

Pour les joints de macle symétriques et à forte densité de coincidence par contre la méthode est moins adaptée que les méthodes interférentielles (franges $\alpha$ ) ou les méthode de diffraction. Ces deux dernières méthodes permettent une bien meilleure précision dans la détermination des translations rigides, et 1 'établis- 
sement non ambigüe des symëtries subsistant dans le joint (voir par exemple les déterminations complètes de $\Sigma 5$ effectuées en utilisant ces deux méthodes (19)). Ces joints ont cependant permis de fournir des objets tests pour la méthode ce qui est précieux lorsque 1'interprêtation devient délicate et dépend de manière assez critique des paramètres expérimentaux. Enfin la MEHR sera dans tous les cas limitēe à un très faible nombre de projections selon des axes d'indice simple (bien que ce nombre augmente assez vite pour des résolutions plus petites) et à des joints de flexion pure ce qui est probablement là sa plus sévēre limitation.

\section{Références}

1 - KRIVANEK 0.L., ISODA S., KOBAYASHI K. Phi1. Mag. 36 (1977) 331

2 - PENISSON J.M., BOURRET A., Phil. Mag. A 40 (1979) 811

3 - ICHINOSE H., ISHINA Y., Phi1. Mag. A 43 (1981) 1253

4 - BOURRET A., RENAULT A., ANSTIS G.R., Chem. Script. 14 (1979) 207

5 - BOURRET A., THIBAULT-DESSEAUX J., D ANTERROCHES C., PENISSON J.M., DE CRECY A., J. of Microscopy (à paraître)

6 - BACMANN J.J., SiLVESTRE G., PETIT M., BOLLMANn W., Phil. Mag. A 43 (1980) 189

7 - KOBVLANSKI A., GOUX C., 1st Inter. Conf. "Soudage et fusion par faisceau d'électrons", CEA p 371 (1971)

8 - DESSEAUX J., RENAULT A., BOURRET A., Phil. Mag. 35 (1977) 357

9 - BOURRET A., DESSEAUX J., Phit. Mag. A 39 (1979) 405

10 - HORNSTRA J., Physica 25 (1959) 409

11 - D'ANTERROCHES C., Thèse (1982) Grenoble

12 - BOURRET A., DESSEAUX J., RENAULT A., Phil. Mag. A 45 (1982) 1

13 - CUNNINGHAM B., AST D., Grain boundaries in semiconductors (1982) p 21 Ed. Pike, Seaker, Leamy-Elsevier

14 - MARKLUND S., Phys. Stat. Sol. (b) 100 (1980) 77

15 - BOURRET A., D'ANTERROCHES C., Colloque CNRS "Semiconducteurs polycristallins" Perpignan (1982)

16 - PENISSON J.M., GRONSKY R., BROSSE J.B., Scripta Met. (ã paraître)

17 - VITEK V., SMITH D.A., POND R.C., Phil. Mag. A 41 (1980) 649

18 - ZEMLIN F., UTtramicroscopy 4 (1979) 241

19 - PAPON A.M., PETIT M., SILVESTRE G., BACMANN J.J., Grain boundaries in semiconductors (1982) p 27. Ed. Pike, Seager, Leamy-Elsevier. 


\section{DISCUSSION}

J.W. CAHN : For the purposes of my paper to follow, it is very important to distinguish between facets, such as in Bacmann's figures in which the boundary normal is different over considerable areas, and your figure 5 , in which there is a regular insertion of single defects into an otherwise ever more regular boundary structure I would consider your beautiful figure to show nature's best attempt to form a planar boundary for that given misorientation.

A. BOURRET : It is true that it is different to describe the structural arrangement of figure 5 as composed of successive facets of two different arrangements. However the boundary is not strictly planar although it appears in this area periodic which is not observed everywhere. As shown in another material (Germanium - Phil. Mag. ref. 9 of our paper) we observed a continuous change when the G.B. normal changes between a pure faceted structure and more or less regular insertion of few other defects in a single "phase".

F. CABANE-BROUTY : Did you sometimes observe segregated atoms?

A. BOURRET : Yes, especially in low angle boundary. These precipitates were recently analyzed as oxygen atoms in Germanium or Silicon matrices (to be published in U1tramicroscopy).

R. BONNET : What is the measuring of the Burgers vector when it is big and dislocations so close? The question arises because of the value of the elastic energy which remains constant after about 10 degrees misorientation?

A. BOURRET : The Burgers vector is a purely geometric description and cannot give the energy. However this description is still interesting in high angle grain boundary, as it can describe the "structural units" which compose the G.B.. Each Burgers vector if correctly chosen can be associated with one structural unit, and most of them can be found as isolated dislocations in very low angle grain boundary or even in the matrix. This is specially true in high stacking fault energy materials where these units remain non dissociated even when they are relatively far from each other. 
L. HOBBS :

It would be fair to point out that there is, dispite your encouraging results on both relaxation and translation measurements, still some concern about the precision of measurements well below the resolution limit of the imaging process. There are, for example, difficulties of lateral coherence and of visibility of partial columns. At this point, it would appear advisable to treat the measurements of relaxation and rigid body translation as somewhat qualitatively, if not schematic.

A. BOURRET : This is true if no simulation is carried out. However we have shown that with proper use of comparison between experimental and simulated images it is possible to obtain details below the resolution limit. This analysis is presently very rarely done, but must be done if quantitative results are needed. Trials on test structures ( $\Sigma 3$ coherent twin) have shown that our present evaluation of the accuracy atom location is correct. 